\title{
Sostenibilidad de sistemas de producción de quinua (Chenopodium quinoa Willd.)
}

\section{Sustainability of quinoa (Chenopodium quinoa Willd.) production systems}

\author{
Rember Pinedo-Taco ${ }^{1 *}$, Luz Gómez-Pando ${ }^{1}$, Alberto Julca-Otiniano ${ }^{1}$ \\ ${ }^{1}$ Universidad Nacional Agraria La Molina, Programa de Posgrado en Agricultura Sustentable. Av. la Molina s/n. Lima, Perú. \\ *Autor de correspondencia: remberpinedo7@hotmail.com
}

Artículo científico recibido: 28 de enero de 2018 aceptado: 06 de julio de 2018

RESUMEN. En los últimos años los altos precios y el aumento de la demanda del mercado nacional e internacional han ocasionado el crecimiento de la superficie cultivada de quinua y la introducción de tecnologías con insumos externos que incrementaron el rendimiento y cambios en el sistema de cultivo. El objetivo de presente estudio fue evaluar los niveles de sustentabilidad económica, ambiental y social de los sistemas de producción de quinua. Se aplicaron encuestas a 92 productores de quinua, estandarizando las respuestas y transformándolas a escala de 1 a 5 , siendo el 5 el mayor valor y 1 el más bajo. Se identificaron cuatro sistemas productivos, teniendo sostenibilidad los sistemas: orgánico, mixto y convencional, con índice de sustentabilidad igual o mayor que el valor medio de la escala. El sistema de producción tradicional no alcanzó el umbral mínimo de 3 en la dimensión económica, por lo que fue calificada como no sustentable por el análisis multidimensional. Para determinar la consistencia interna se utilizó el método factorial de Análisis de Correspondencia Múltiple (ACM) con valores de 0.814 y 0.797 de alfa de Crombash que le confieren confiabilidad al análisis.

Palabras clave: Análisis multidimensional, indicador, índice de sostenibilidad, producción sostenible

ABSTRACT. In recent years, high prices and increased demand from the domestic and international markets have led to an increase in the quinoa-growing area and the introduction of technologies with external inputs that increased yields and resulted in changes in the cropping system. The objective of this study was to evaluate the levels of economic, environmental and social sustainability of the quinoa production systems. Surveys were applied to 92 quinoa producers, standardizing the responses by transforming them to a scale of 1 to 5 , with 5 being the highest sustainability value and 1 being the lowest. Four production systems were identified, with the systems having sustainability being the organic, mixed and conventional ones, with a sustainability index equal to or greater than the average value of the scale. The traditional production system did not reach the minimum threshold of 3 in the economic dimension, so it was categorized as non-sustainable by the multidimensional analysis. In order to determine internal consistency, the Multiple Correspondence Analysis (MCA) factorial method was used, with Cronbach's alpha values of 0.814 and 0.797 that confer reliability to the analysis.

Key words: Multidimensional analysis, indicator, sustainability index, sustainable production

\section{INTRODUCCIÓN}

En los últimos años en Perú, el cultivo de quinua se incrementó por la alta demanda del mercado y los altos precios (Vargas et al. 2015). El área cultivada incremento, y se intrudujeron nuevas prácticas culturales e insumos (Soto et al. 2012). En el 2014 la producción nacional de quinua fue de 114 $000 \mathrm{t}$, lo que representa un crecimiento del $119 \%$ con relación al 2013 (MINAGRI 2015). En el departamento de Ayacucho, en los últimos 10 años el área cultivada de quinua aumentó de 244 a 2536 ha, lo que representa un incremento del 939\% (DRAA 2014); en tanto que en la campaña 2014/15, el área sembrada a nivel departamental alcanzó las 11 115 ha (DRAA 2015). Sembrándose en los valles interandinos la quinua en monocultivo, con predominio del sistema de producción de uso intensivo de 
suelos, con mecanización agrícola, fertilizantes sintéticos y pesticidas, variedades mejoradas de color blanco que desplazan a los genotipos locales de color, así como la disminución de la producción de otros cultivos tradicionales (IICA 2015).

La quinua se siembra en cuatro sistemas de producción: convencional, con alta dependencia de insumos externos, servicios de mano de obra y maquinaria (Mercado y Ubillus 2017); tradicional, con bajos índices de producción y productividad, en áreas reducidas con economías de subsistencia (Bolívar 2011); mixtos o alternativos con uso racional de agroquímicos y manejo responsable de los medios de producción (IICA 2015); y orgánico, con asistencia técnica, para mercados de exportación (Campos et al. 2017). En el contexto internacional, para el 2030 se deben de tener sistemas de producción sostenibles, con prácticas agrícolas que incrementen la producción y productividad, económicamente viables, ecológicamente adecuados y, cultural y socialmente aceptables; pero, al mismo tiempo conserven los ecosistemas (Sarandón 2002, ONU 2015).

La sostenibilidad es un concepto complejo y multidireccional, que hace difícil su evaluación (Benítez et al. 2016), con propuestas metodológicas para medirla (Sarandón 2002). En general, la mayoría proponen, la evaluación de las dimensiones: ecológica, económica y social o sociocultural (Blandi et al. 2015). Para evaluar la sustentabilidad de cada dimensión señalada, hay métodos de análisis multicriterio por medio de la evaluación de un conjunto de indicadores para conocer los puntos críticos de la sustentabilidad de los agroecosistemas (Meza y Julca 2015). Por lo anterior, el objetivo fue evaluar la sustentabilidad de los sistemas de producción de quinua del distrito de Chiara-Ayacucho.

\section{MATERIALES Y MÉTODOS}

El estudio se realizó en las comunidades de Kishuarcancha, Manallasac y Sachabamba del distrito de Chiara provincia de Huamanga, Ayacucho, ubicado a $3516 \mathrm{msnm}$, en las coordenadas geográficas $13^{\circ} 16^{\prime} 26.3^{\prime \prime}$ LS y $74^{\circ} 12^{\prime} 21.9^{\prime \prime}$ LO. La vegetación de la zona de estudio es bosque húmedo Montano Sub tropical (bh-MS), con baja humedad relativa en el sector de la variante, con valores promedio anual entre 40 y $60 \%$. La precipitación anual oscila entre 650 y $690 \mathrm{~mm}$, con temperaturas entre 9 y $11{ }^{\circ} \mathrm{C}$, máxima de 20.7 y mínima de 2.2 ${ }^{\circ} \mathrm{C}$ (GOREA 2012). La zona de estudio tiene una población de 460 productores que cultivan quinua, de la cual se tomó una muestra de 92 productores; mediante muestreo sistemático y de manera proporcional se estableció la distribución de productores por comunidad (Barreto 2015, Meza y Julca 2015).

Para el análisis multicriterio propuesto por Sarandón (2002), se utilizaron variables e indicadores previamente validados en un taller realizado en Chiara con fines de análisis, 10 indicadores y 24 subindicadores se agruparon en la dimensión económica, ambiental y social (Tabla 1). Los datos de las encuestas se estandarizaron para cada indicador mediante la transformación a una escala de 1 a 5 ; siendo 5 el mayor valor de sustentabilidad y 1 el más bajo. Para determinar la situación actual de los indicadores, independientemente de las unidades medidas originalmente, los valores de cada indicador se expresaron en algún valor de la escala (Sarandón y Flores 2014). Luego, se establecieron ponderaciones de acuerdo al grado de importancia y peso de cada indicador (Sarandón et al. 2006).

La consistencia interna de las escalas utilizadas en cada variable/indicador y el nivel de similitud de estas, se determinó con el Análisis de Correspondencia Múltiple (ACM) con escalamiento óptimo, para describir en un espacio de pocas dimensiones la estructura de asociaciones entre variables ordinales, así como las similitudes y diferencias entre los casos (Benítez et al. 2016). Para hallar la consistencia interna de los datos se consideró un Alfa de Cronbach no menor de 0.7, ya que valores menores revelan una débil relación entre las variables analizadas (Gonzales y Pazmiño 2015).

El valor de los Indicadores Económicos (IK), Indicadores Ambientales (IA) e Indicadores Sociales (IS), se determinaron con la suma algebraica de sus respectivos indicadores, que se multiplicaron por su factor de ponderación, con las siguientes rela- 
Tabla 1. Indicadores para medir la sustentabilidad de sistemas de producción de quinua.

\begin{tabular}{lll}
\hline Dimensión económica (IK) & Dimensión ambiental (IA) & Dimensión social (IS) \\
\hline A.- Rentabilidad & A.- Conservación de la vida de suelo & A.- Satisfacción de las necesidades básicas \\
A1.- Superficie cultivada & A1.- Rotación de cultivos & A1.- Vivienda. \\
A2.- Productividad & A2.- Diversificación de cultivos & A2.- Nivel de educación \\
A3.- Incidencia de Plagas & A3.- Incorporación de materia orgánica & A3.- Acceso a salud y cobertura \\
B.- Ingreso económico & B.- Riesgo de erosión & A4.- Servicios. \\
B1.- Ingreso Neto Mensual & B1.- Preparación de terreno & B.-Aceptabilidad sistema de producción \\
-Ingreso por cultivo principal & B2.- Sistema de producción & B1.- Nivel de satisfacción del productor \\
-Ingreso cultivo secundario & B3.- Pendiente predominante & B2.- Sistema de producción \\
-Ingreso otras actividades & B4.- Cobertura vegetal & C.- Integración social \\
C.- Riesgo económico & C.- Manejo de la Biodiversidad & C1.- Nivel de integración social \\
C1.- Diversificación para la venta & C1.- Conservación de variedades de quinua & D.- Asistencia técnica y capacitación \\
C2.- No canales comercialización & C2.- Gestión de plagas & D1.- Nivel asistencia técnica y capacitación \\
C3.- Dependencia de insumos: & C3.- Manejo semilla de calidad & \\
\hline A, C, Dencion
\end{tabular}

A, B, C, D = Variables; A1, B1, C1, D1 = Sub indicadores.

ciones matemáticas: $\mathbb{I K}=[2((\mathrm{~A} 1+\mathrm{A} 2+\mathrm{A} 3) / 3)+$ $\mathrm{B}+(\mathrm{C} 1+\mathrm{C} 2+\mathrm{C} 3) / 3] / 4 ; \mathrm{IA}=[2((\mathrm{~A} 1+$ $\mathrm{A} 2+\mathrm{A} 3+\mathrm{A} 4) / 4)+(\mathrm{B} 1+\mathrm{B} 2+\mathrm{B} 3) / 3+(\mathrm{C} 1$ $+\mathrm{C} 2) / 2] / 4$ y el IS $=[2((\mathrm{~A} 1+\mathrm{A} 2+\mathrm{A} 3+\mathrm{A} 4) 4)$ $+\mathrm{B} 1+\mathrm{C}+\mathrm{D}] / 5$. El índice de sustentabilidad general (ISG) se estimó con la relación matemática $I S G=(I K+I A+I S) / 3$. El valor umbral o mínimo que debe alcanzar el ISG para considerar que la producción de quinua, bajo los sistemas de producción identificados, es sustentable debe ser igual o mayor que el valor medio de la escala, es decir, 3 para las tres dimensiones consideradas (Sarandón et al. 2006). A cada valor del ISG se le realizó la estratificación de sustentabilidad con un gradiente ascendente para los procesos menos sustentables (menor a 3) y más sustentables (mayor o igual a 3), como se indica a continuación: muy crítica ( 0 a 1.99), crítica (2 a 2.99), débil (3 a 3.9), media (4 a 4.99), y alta (mayor a 5).

\section{RESULTADOS}

El análisis de consistencia interna para las 24 variables, tuvo valores de 0.814 y 0.797 alfa de Cronbach, lo que le confiere buena confiabilidad al análisis de consistencia y pertinencia interna de los indicadores y la escala utilizada. Este análisis determinó los niveles de asociación, relaciones de dependencia y semejanza de los productores estudiados. En la Figura 1, se observan los productores tradicionales identificados con los números $53,54,73$,
74 , los cuales tienen baja dependencia de insumos externos, y bajos niveles de rentabilidad y productividad. Mientras que los productores con sistemas orgánicos, mixtos y convencionales se encuentran cerca de la dimensión 2; la cual es la más idónea y representativa para definir la sostenibilidad de los sistemas de producción de quinua en el distrito de Chiara por su mayor asociación entre variables.

El análisis de sostenibilidad económica (IK), determinó que el sistema de producción orgánico tiene un valor de 3.08, el cual es el mayor valor; mientras que el valor más bajo y menos sostenible lo tuvo el sistema tradicional con IK de 2.15 (Tabla 2). Los indicadores que afectan la sostenibilidad económica de los productores tradicionales son la rentabilidad, el ingreso neto mensual y el riesgo económico. Para determinar los puntos críticos y la sostenibilidad de los sistemas de producción de quinua en relación con los indicadores económicos (IK), se realizó un diagrama tipo ameba (Figura 2). Se observa que el sistema tradicional es el menos dependiente de insumos externos. Pero presenta los puntos críticos siguientes: pequeñas superficies de siembra, baja productividad, dificultades de acceso a mercados, lo que afecta la rentabilidad. El ingreso neto mensual para el sistema de producción tradicional fluctúo entre 149.40 y USD 299.11, mientras que agricultores con producción orgánica, mixta tienen ingresos entre 299.11 y USD 1 496.71.

Los resultados del Indicador Ambiental (IA), para los cuatro sistemas muestra valores superiores 


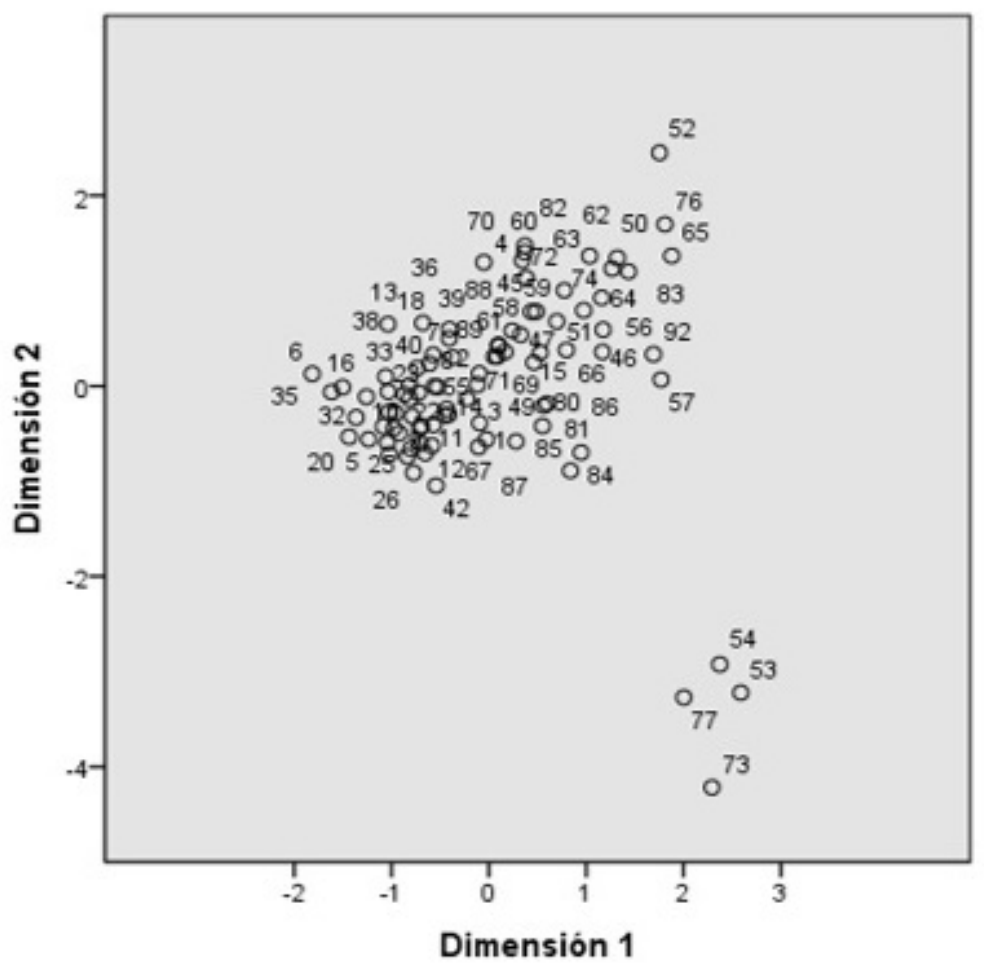

Figura 1. Puntos de objeto etiquetados por número de caso. Los números 53, 54,73 y 74 corresponden a productores tradicionales.

Tabla 2. Valores de indicadores de sustentabilidad de sistemas de producción de quinua.

\begin{tabular}{cccccccccccccc}
\hline Sistema & RNT & INM & RIE & IK & CVS & RDE & MAB & IA & SNB & ASP & NIS & NAT & IS \\
\hline Tradicional & 2.67 & 1.25 & 2.00 & $2.15^{*}$ & 4.25 & 3.50 & 2.75 & $4.19^{* *}$ & 3.50 & 3.75 & 2.50 & 3.25 & $3.30^{* *}$ \\
Orgánico & 3.56 & 2.90 & 2.3 & $3.08^{* *}$ & 3.75 & 3.24 & 3.01 & $3.44^{* *}$ & 3.78 & 3.43 & 3.45 & 3.10 & $3.51^{* *}$ \\
Mixto & 3.59 & 2.84 & 2.14 & $3.04^{* *}$ & 3.69 & 3.41 & 2.67 & $3.36^{* *}$ & 3.59 & 2.63 & 3.47 & 3.19 & $3.29^{* *}$ \\
Convencional & 3.67 & 3.00 & 2.12 & $3.11^{* *}$ & 3.74 & 3.61 & 2.36 & $3.36^{* *}$ & 3.56 & 2.18 & 3.00 & 3.00 & $3.06^{* *}$
\end{tabular}

* No sustentable con valor $<3,{ }^{* *}$ Sustentable con valor $\geq 3$. Indicador Económico (IK): Rentabilidad (RNT), Ingreso Neto Mensual (INM), Riesgo Económico (RIE). Indicador Ambiental (IA): Conservación Vida del Suelo (CVS); Riesgo de Erosión (RDE); Manejo de la Biodiversidad (MAB). Indicador Social (IS): Satisfacción de las Necesidades Básicas (SNB), aceptabilidad del sistema de producción (ASP), Nivel de asistencia técnica y capacitación (NAT).

a 3; siendo el sistema tradicional el más sustentable con IA de 4.19. Lo que se debe a los valores de los indicadores de Conservación Vida del Suelo (CVS) y Riesgo de Erosión (RDE) con valores de 4.25 y 3.50 , respectivamente; los cuales fueron los mayores, lo que fue favorecido por la forma de preparación del suelo y la cobertura vegetal; mientras que en los demás sistemas tienen valores menores (Tabla 2). En la Figura 3, se observa que los indicadores críticos del sistema convencional, mixto y orgánico son la diversificación de cultivos por la práctica de monocultivo, la preparación del terreno por el uso intensivo de maquinaria, agrícola y la gestión de plagas con alta dependencia de agroquímicos en el sistema convencional, mientras que en el sistema orgánico tiene dependencia de bioinsecticidas. El sistema tradicional tiene los mayores valores, los cuales se acercan al umbral óptimo por el uso de materia orgánica y la rotación de cultivos, manejo de cobertura vegetal y la preparación de terreno con prácticas poco intensivas.

El Indicador Social (IS) en los cuatro sis- 


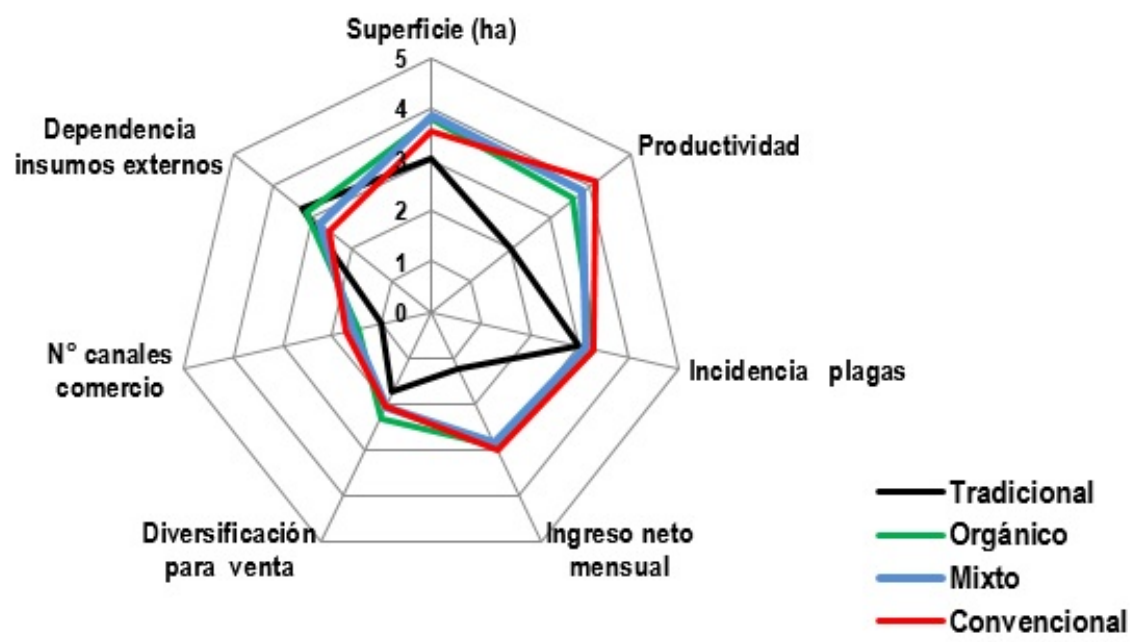

Figura 2. Diagrama de indicadores de niveles de sustentabilidad económica de sistemas de producción de quinua.

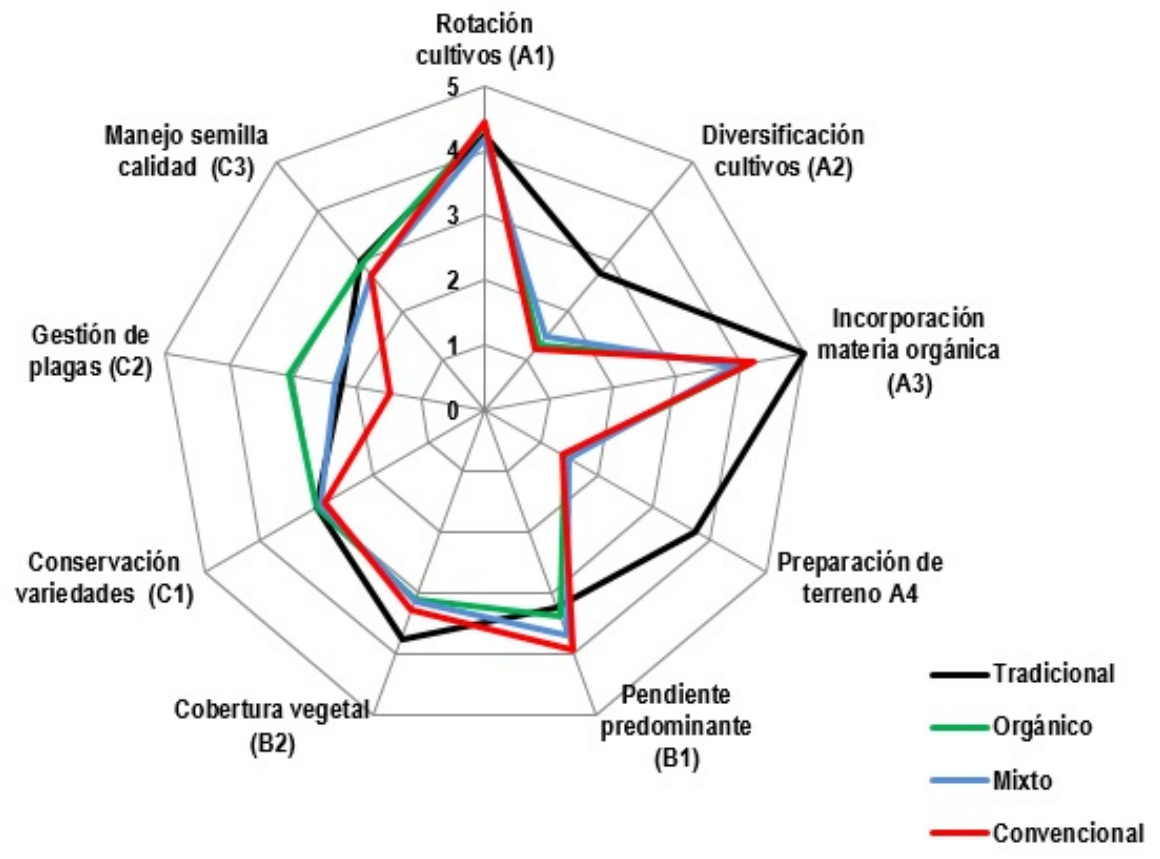

Figura 3. Diagrama de valores de indicadores de sustentabilidad ambiental de sistemas de producción de quinua en Chiara, Ayacucho.

temas, tuvo valores mayores a 3, lo que indica que son sustentables. En el sistema tradicional el indicador de NIS es de 2.50, valor crítico que refleja bajos niveles de organización y escasa interacción participativa, lo que dificulta la adopción de tec- nologías. De acuerdo con la percepción de los productores, los sistemas que pueden mejorar la convivencia y equilibrio socioeconómicos son los sistemas de producción tradicional, orgánica, y mixta (Figura 4). Estos sistemas incorporan tecnologías 


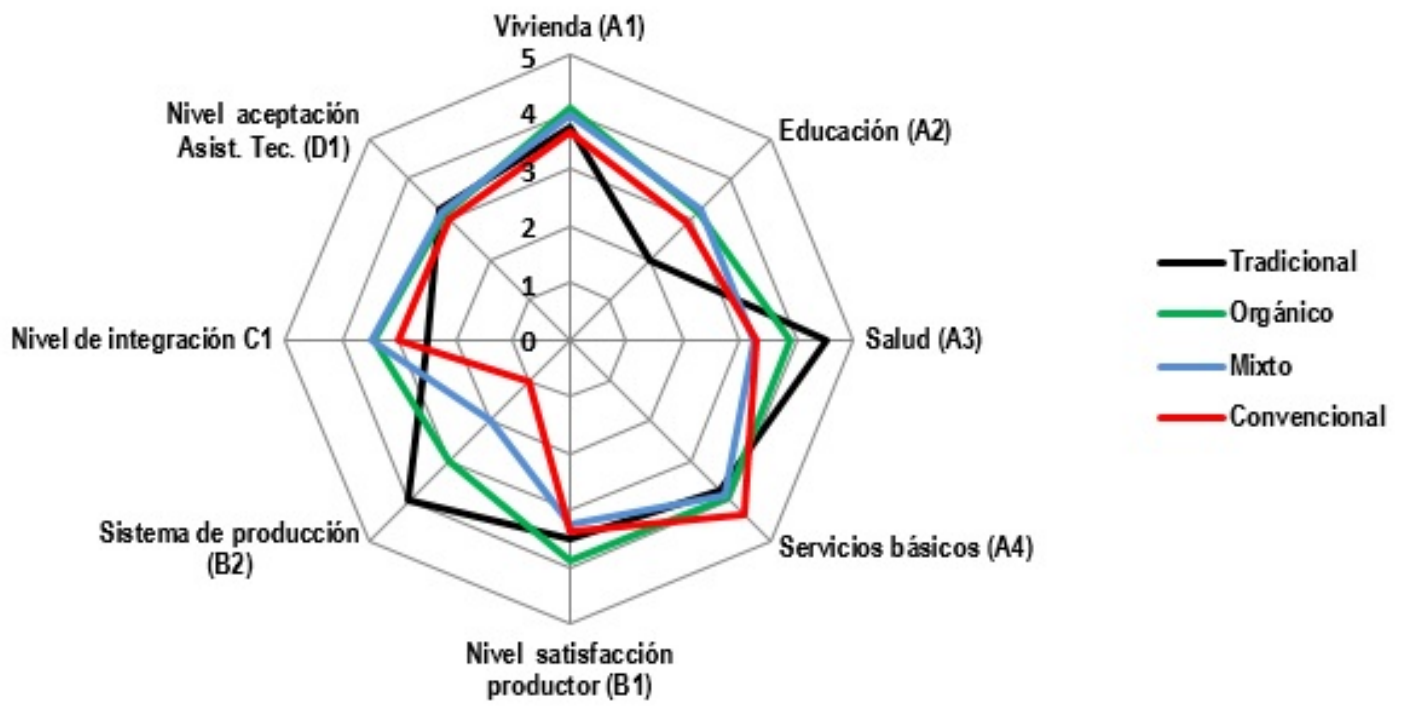

Figura 4. Diagrama valores de indicadores de sustentabilidad social de sistemas de producción de quinua.

tradicionales, son menos dependientes de insumos externos y viven en los poblados que están cerca de sus parcelas de producción; mientras que los productores convencionales emplean tecnología externa, contratan mano de obra fuera de la comunidad, y en su gran mayoría son arrendatarios y viven en las ciudades.

Los cuatro sistemas de producción son sostenibles con valores de ISG de 3.21, 3.34, 3.23 y 3.18 , respectivamente (Figura 5). Para el IK, el sistema tradicional tiene un valor menor a 3 , lo que indica que no cumple con el requisito de sostenibilidad de acuerdo con el análisis multicriterio utilizado. Los resultados independientes de ISG (Figura 6) para los sistemas de producción orgánico, mixto y convencional tienen sustentabilidad débil, los valores de IK, IA e IS son un poco mayores del umbral mínimo aceptable de sostenibilidad, por lo que se requiere implementar medidas para mejorar su valoración, ya que cualquier adversidad en aspectos económicos, ambientales y sociales pueden afectar la sostenibilidad. El sistema tradicional tiene posibilidades de superar el umbral mínimo de sostenibilidad y posiblemente escalar hacia valores óptimos; pero requieren asistencia técnica y políticas que favorezcan su estabilidad.

\section{DISCUSIÓN}

En el análisis de consistencia interna de las variables estudiadas, tuvo valores de 0.814 y 0.797 alfa de Cronbach, lo que indica buena consistencia interna de los datos y confiabilidad para definir las características y sostenibilidad de los sistemas de producción de quinua. Al respecto Gonzales y Pazmiño (2015), afirman que un valor del alfa de Cronbach, entre 0.70 y 0.90 indica buena consistencia interna debido a que las variables que conforman las dimensiones están correlacionadas y son interdependientes, lo que garantiza la pertinencia del análisis de correspondencias y permite clasificar los sistemas de producción.

En el análisis comparativo del Indicador Económico (IK), muestra que el sistema tradicional tiene el valor más bajo y menos sostenible de los sistemas estudiados (Tabla 2), con valor IK de 2.15 que indica sustentabilidad crítica, por los bajos ingresos económicos, condiciones económicas precarias y subsistencia. Al respecto Meza y Julca (2015) reportan IK similares para el sistema de producción tradicional. Los sistemas de mayor productividad son el orgánico, mixto y el convencional que en algunos casos superan las $3 \mathrm{t} \mathrm{ha}^{-1}$ con ingresos men- 


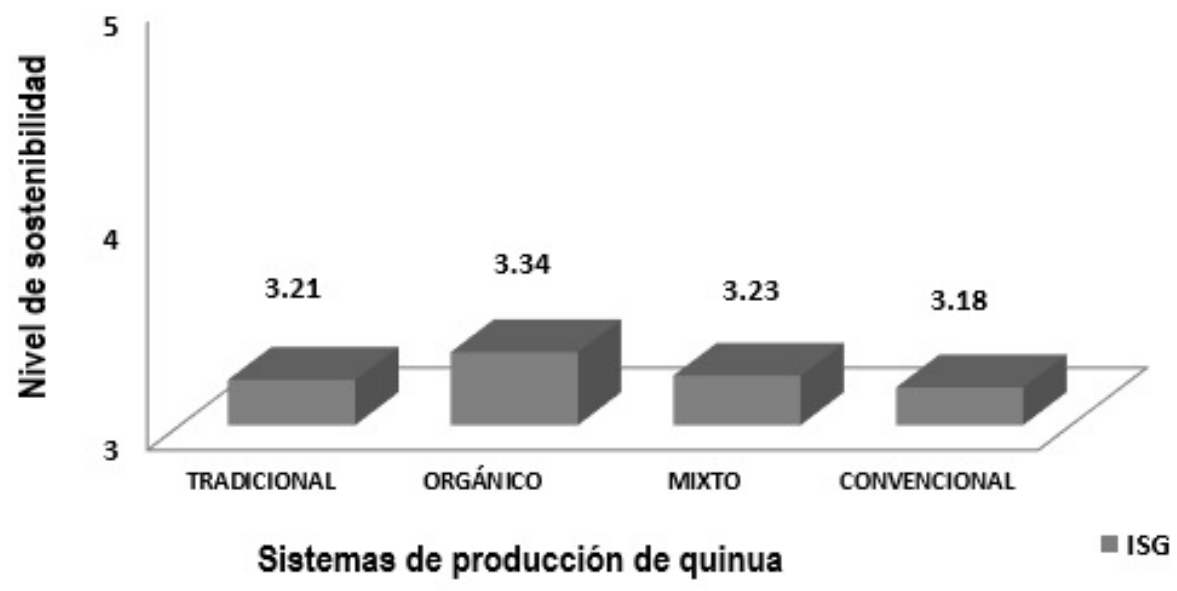

Figura 5. Índice de sustentabibilidad general (ISG) de los sistemas de producción de quinua.

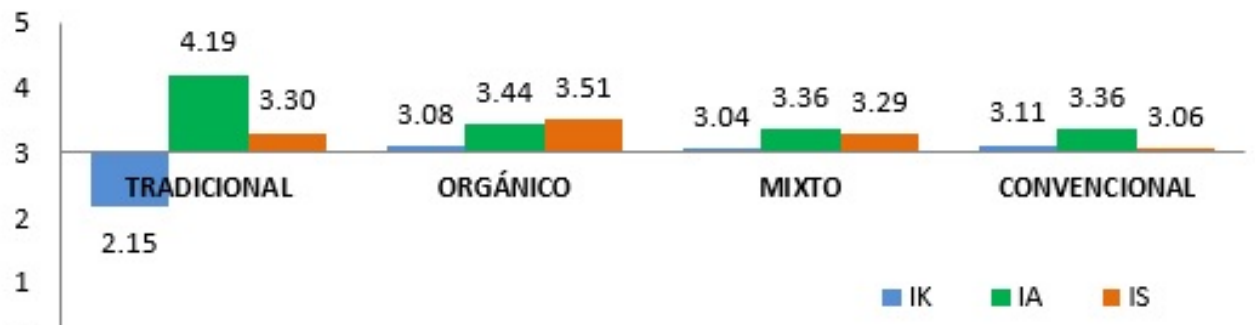

Sistemas de producción

Figura 6. Indicadores de sustentabilidad económica (IK) ambiental (IA) y social (IS) en sistemas de producción de quinua.

suales que oscilán entre 299.10 y USD 1 496.71; mientras que en el sistema tradicional, la productividad no alcanza una tonelada, lo que reduce el ingreso mensual. Al respecto, Mercado y Ubillus (2017), indican que la rentabilidad de la producción orgánica de quinua es mayor que la quinua producida en forma tradicional. Debido a la diferencia de precio y el acceso al mercado, mientras que los pequeños y medianos productores reciben un menor precio, por no acceder al mercado de los medianos y grandes agricultores que tienen certificación orgánica y contrato con las empresas que determinán el precio de la quinua orgánica.

Los sistemas orgánico, mixto y convencional tienen valores de IK mayor a 3, pero se encuentran en nivel de sustentabilidad bajol; por lo que requieren incorporar tecnologías apropiadas para incrementar la productividad y rentabilidad del cultivo. Además de hacer uso de variedades mejoradas, reducir los niveles de daño económico ocasionado por plagas, y mejorar el manejo integrado del cultivo. Al respecto, Pinedo et al. (2017) reportan que sólo el $5.4 \%$ de los productores de los sistemas de producción orgánico y convencional usan semilla certificada, mientras que el sistema tradicional usan variedades nativas. El sistema tradicional, puede mejorar sus niveles de rentabilidad si adopta el uso semilla mejorada con variedades que demanda el mercado. Pero esta condición perjudicaría al valor de su IA, debido a que el modelo de producción tiende al monocultivo y se desplazarían las variedades nativas (Vargas et al. 2015). 
En el diagrama de la Figura 2, se observan que el indicador incidencia de plagas de los cuatro sistemas de producción apenas supera el valor de 3 , lo que califica a los sistemas de producción como sustentabilidad débil; siendo lo ideal que los sistemas mixto y convencional con alta tecnología, tengan valores cercanos a 5 . El principal factor que influye en la mayor incidencia de plagas en la producción orgánica, mixta y convencional; es el monocultivo. Al respecto, Sarandón y flores (2014) reportan que los modelos de producción que practican el monocultivo con una sola especie tienen mayor problema de plagas; mientras que el policultivo tiene mayor estabilidad frente a los problemas de plagas. En el monocultivo la presión de plagas es mayor que en sistemas diversificados, lo que afecta su rentabilidad (Medrano et al. 2011, Soto et al. 2012). Siendo los mayores problemas de plagas de la quinua las larvas del complejo Eurysacca sp, el mildiu (Peronospora variabilis), las palomas y los cernícalos (Pinedo et al. 2017). Al respecto, Robles et al. (2003) indican que el daño por aves como la paloma rabiblanca (Zenaida auriculata) pueden ocasionar pérdidas económicas de hasta el $60 \%$ de la producción de quinua.

Los cuatro sistemas fueron sostenibles para el IA, los sistemas de producción orgánica, mixta y convencional tienen sustentabilidad débil, mientras que el sistema de producción tradicional tiene sustentabilidad media. Lo que coincide con Barreto et al (2015) y Meza y Julca (2015) quienes reportan sostenibilidad intermedia para los sistemas de producción tradicional. El valor IA de 4.19 del sistema de producción tradicional se aproxima más a la situación ideal, pero con valores de 2.75 en el manejo de la biodiversidad, lo que indica desplazamiento y perdida de ecotipos locales de quinua por el cambio de variedades mejoradas. Al respecto, Vargas et al. (2015), indican que las políticas de fomento de cultivo y la demanda del mercado han desplazado las variedades nativas y los sistemas de producción diversificados. Mientras que Pinedo et al. (2017) señalan que los sistemas de producción tradicionales de quinua tienden a la perdida de variedades locales por el reemplazo con variedades de alta demanda en el mercado.

El manejo del recurso suelo es un factor crítico en todos los sistemas. En el indicador riesgo de erosión que comprende los subindicadores pendiente predominante y rotación de cultivos, los sistemas tradicionales, orgánicos, mixtos y convencionales tienen valores de $3.50,3.24,3.41$ y 3.61 , respectivamente. Al respecto, Pinedo et al. (2017) indican que la pendiente predominante de los suelos en la zona de estudio varía de 5 a 30\%, lo que facilita el uso de maquinaria agrícola en la preparación de suelos. Con respecto a la rotación de cultivos, el 39\% de los productores tradicionales cambian de cultivo cada año o dos años, mientras que en los sistemas convencionales sólo el 15\% realizan rotación de cultivos (Pinedo et al 2017). Sobre lo mismo el IICA (2015) indica que los sistemas orgánicos realizan rotación, debido a las normas técnicas y los acuerdos con empresas exportadoras; mientras que los sistemas convencionales con monocultivos rotan cada tres años; entretanto en los sistemas tradicionales, la parcela suele descansar de uno a tres años (Soto el al. 2012).

El empleo de materia orgánica es mayor en el sistema tradicional, lo que tiene incidencia en el mayor valor en el indicador de CVS, mientras que los otros sistemas presentan valores intermedios. Al respecto, Barreto et al. (2015) en la evaluación de sostenibilidad ecológica, indican que el $53.1 \%$ de los productores de sistemas de producción tradicional, consideran que la CVS se puede lograr con la aplicación periódica de materia orgánica.

El valor del IS es sostenible para los cuatro sistemas de producción, pero con valores entre 3.00 y 3.99, que indica sostenibilidad débil. El sistema tradicional en el indicador Nivel de Integración Social (NIS $=2.50$ ), presenta el valor más bajo, debido al debilitamiento de su sistema organizativo. Al respecto el IICA (2015), señala que en la zona altoandina del Perú, aproximadamente el $80 \%$ de los productores desarrollan su actividad productiva en forma individual y el $20 \%$ está asociado con alguna organización de productores. El sistema orgánico presenta el mayor NIS, debido a que están organizados en función de un mercado de destino, y su- 
pervisados por un organismo certificador que les da soporte (Pinedo et al. 2017). Al respecto, Candelaria, et al. (2014), consideran que la dimensión social es prioritaria en el desarrollo de alternativas de sustentabilidad, con particular importancia en el proceso de organización de los productores como mecanismo para innovar los sistemas de producción, mediante la adopción, implementación y adecuación de tecnologías.

En el diagrama de la Figura 4, en el indicador Nivel de Asistencia Técnica (NAT) el sistema de producción tradicional muestra el mayor valor frente a los otros sistemas, pero esto no se ve reflejado en mejores condiciones socioeconómicas del productor de quinua. Si bien hay presencia del estado y entidades privadas los procesos de fortalecimiento de capacidades y metodologías utilizadas no se adecuan a los sistemas de producción tradicional, con la consecuente pérdida de los conocimientos y prácticas ancestrales. Para los sistemas orgánicos, mixto y convencional el escenario parece ser más favorable, debido a que el agricultor tiene mayor atención en aspectos técnico-productivos y comercialización por las instituciones proveedoras de asistencia técnica (IICA 2015, Mercado y Ubillus 2017). Los cuatro sistemas de producción alcanzaron valores de ISG mayores a 3. Pero los del sistema tradicional tienen un IK menor de 3, lo que indica que no es sostenible (Sarandón 2002); además los bajos niveles de rentabilidad asociadas a la baja productividad y la escasa capacidad de negociación colectiva, causan que su sustentabilidad sea crítica. Al respecto, Pinedo et al. (2017) encontraron que el $4.35 \%$ son productores tradicionales conservacionistas, con bajos niveles de rentabilidad y NIS. Lo que indica que aúnque alcancen indicadores IK e IS sostenibles, el factor económico debilita al sistema. Al respecto, Gómez et al. (2015) y Sarandón et al. (2006) indican que un sistema de producción no es sostenible, cuando uno de los indicadores tiene valores por debajo del umbral mínimo de sostenibilidad.
En los sistemas orgánico, mixto y convencional el ISG les confiere sustentabilidad en estatus débil, por lo que cualquier cambio en mercado, clima o financiamiento pueden afectarlos; lo que indica que la tecnificación de los agroecosistemas altoandinos no garantiza la producción estable a largo plazo. La introducción de nuevos sistemas de producción de quinua, ha causado cambios en el manejo del cultivo tradicional; como mayor mecanización del cultivo en sus diferentes fases, reducción de superficie cultivada con variedades locales, e incremento de la superficie cultivada con variedades preferidas en el mercado, mayor empleo de insumos agrícolas y menor consumo de la quinua como alimento familiar. Al respecto Barrientos (2017) reporta que la introducción o el establecimiento de nuevos sistemas de producción no garantizan la sustentabilidad del cultivo de quinua. Mientras que Vargas et al. (2016) indican que actualmente prevalece una tendencia de la sustitución de los modos de producción tradicionales. En tanto que Blandi et al. (2015) y Barrezueta (2015), indican que la mayor tecnificación de los sistemas de producción conduce a sistemas menos sustentables debido al uso inadecuado de recursos no renovables.

\section{CONCLUSIONES}

El análisis de Correspondencia Múltiple validó la consistencia interna de la escala ordinal de los subindicadores y agrupó a los productores por semejanzas y proximidades en dos dimensiones, la primera agrupa a los sistemas de producción orgánico, mixto y convencional, con predominio del monocultivo y la segunda al sistema de producción tradicional diversificada. Los sistemas de producción orgánico, mixto y convencional son sostenibles, con valores mayores al umbral mínimo establecido. El sistema orgánico tiene posibilidades de mantenerse por encima del umbral mínimo de sostenibilidad, siempre que se mejoren los indicadores evaluados. 


\section{LITERATURA CITADA}

Barrientos E, Carevic F, De-Latorre J (2017) La sustentabilidad del altiplano sur de Bolivia y su relación con la ampliación de superficies de cultivo de quinua. Idesia 35: 7-15

Barreto J, Canto M, Julca A (2015) Sostenibilidad ecológica de la producción agropecuaria tradicional de Carhuáz, Áncash, Perú. Aporte Santiaguino 8: 219-228.

Barrezueta S (2015) Introducción a la sostenibilidad agraria: Con enfoque de sistemas e indicadores. Universidad Técnica de Machala. Primera edición. Ecuador. 69p.

Benítez G, Alvarado G, Ortíz G, Sangabriel W, Lara A (2016) Evaluación rápida de la sostenibilidad en la región de la laguna de Cuyutlán, Colima, México. Interciencia 41: 588-595.

Blandi ML, Sarandón SJ, Flores CC, Veiga I (2015) Evaluación de la sustentabilidad de la incorporación del cultivo bajo cubierta en la horticultura platense. Revista de la Facultad de Agronomía 114: 251-264.

Bolívar H (2011) Metodologías e indicadores de evaluación de sistemas agrícolas hacia el desarrollo sostenible. CICAG 8: 1-18.

Candelaria B, Ruiz O, Pérez P, Gallardo F, Vargas L, Martínez A, Flota-Bañuelos C (2014) Sustentabilidad de los agroecosistemas de la microcuenca Paso de Ovejas 1. Cuadernos de Desarrollo Rural 11: 87-104.

Campos A, Cabrera P, Pérez C, Laura C (2017) Tendencia del mercado y la producción de los productos orgánicos en el Perú. Revista Investigación Altoandina 19: 427-431

DRAA (2014) Series históricas de siembras y cosechas. Dirección Regional Agraria Ayacucho. http://www.a groayacucho.gob.pe/estadisticas. Fecha de consulta: 27 de diciembre de 2017.

DRAA (2015) Series históricas de siembras y cosechas. Dirección Regional Agraria Ayacucho. http://www.a groayacucho.gob.pe/estadisticas. Fecha de consulta: 30 de noviembre de 2017.

Gómez LF, Ríos-Osorio LA, Eschenhagen ML (2015) Sostenibilidad en agroecología. Revista UDCA Actualidad \& Divulgación Científica 18: 329 - 337.

González JA, Pazmiño, M (2015) Cálculo e interpretación del Alfa de Cronbach para el caso de validación de la consistencia interna de un cuestionario, con dos posibles escalas tipo Likert. Revista Publicando 2: $62-77$.

GOREA (2012) Informe temático Zonas de vida, Ayacucho. Gobierno Regional de Ayacucho. Gerencia Regional de Recursos Naturales y Gestión del Medio Ambiente. Perú. 58p

IICA (2015) El mercado y la producción de quinua en el Perú. Instituto Interamericano de Cooperación para la Agricultura. Lima. Perú. 172p.

Medrano AM, Torrico JC, Fortanelli J (2011) Análisis de la sostenibilidad de la producción de quinua (Chenopodium quinoa Willd.) en el intersalar boliviano. CienciAgro 2: 303-312

Mercado W, Ubillus K (2017) Characterization of producers and quinoa supply chains in the Peruvian regions of Puno and Junin. Scientia Agropecuaria 8: 251-265

Merma I, Julca A (2012) Caracterización y evaluación de la sustentabilidad de fincas en Alto Urubamba, Cusco, Perú. Ecología Aplicada 11: 1-11.

Meza Y, Julca A (2015) Sustentabilidad de los sistemas de cultivo con yuca (Manihot esculenta Crantz) en la subcuenca de Santa Teresa, Cusco. Ecología Aplicada 14: 55-63. 
MINAGRI (2015) Quinua peruana, situación actual y perspectivas en el mercado nacional e internacional. Ministerio de Agricultura y Riego. Lima, Perú. 67p.

ONU (2015) Open Working Group proposal for Sustainable Development Goals. United Nations Organization. Full report of the of the General Assembly on is issued as document A/68/970, available at http://undocs.org/A/68/970

Pinedo R, Gómez L, Julca A (2017) Caracterización de los sistemas de producción de quinua (Chenopodium quinoa Willd) en el distrito de Chiara, Ayacucho. Aporte Santiaguino 10: 351-364.

Robles J, Jacobsen SE, Rasmussen C, Otazu V, Mandujano J (2003) Plagas de aves en quinua (Chenopodium quinoa Willd.) y medidas de control en el Perú central. Revista Entomológica del Perú 43: 147-151.

Sarandón SJ (2002) El desarrollo y uso de indicadores para evaluar la sustentabilidad de los agroecosistemas. En: Sarandón SJ (ed.). Agroecología: El camino para una agricultura sustentable. Ediciones Científicas Americanas. La Plata, Argentina. pp. 393-414

Sarandón SJ, Zuluaga MS, Cieza R, Gómez C, Janjetic L, Negrete E (2006) Evaluación de la sustentabilidad de sistemas agrícolas de fincas en Misiones, Argentina, mediante el uso de indicadores. Revista Agroecología 1: $19-28$.

Sarandón SJ, Flores CC (2014) Agroecología: bases teóricas para el diseño y manejo de Agroecosistemas sustentables. $1^{\text {a }}$ Ed. Universidad Nacional de La Plata. Argentina. 466p.

Soto J, Valdivia E, Valdivia R, Cuadros A, Bravo R (2012) Descripción de sistemas de rotación de cultivos en parcelas de producción de quinua en cuatro zonas (siete distritos) del altiplano peruano. CienciAgro 2: $391-402$

Vargas HDE, Martí B, Araca L, Vargas W, Vargas R (2015) Agrobiodiversidad y economía de la quinua (Chenopodium quinoa) en comunidades aymaras de la cuenca del Titicaca. Revista Idesia 33: 81-87. 
